\title{
Evaluation of Uterocervical Angle and Cervical Length as Predictors of Spontaneous Preterm Birth
}

\author{
Pramod Kumar Singh ${ }^{1}$ (i) Resham Srivastava $^{1} \quad$ Ishan Kumar $^{10} \quad$ Sangeeta Rai ${ }^{2} \quad$ Saurabh Pandey ${ }^{1}$ \\ Ram C. Shukla ${ }^{1}$ Ashish Verma ${ }^{1}$ \\ ${ }^{1}$ Department of Radiodiagnosis and Imaging, IMS, Banaras Hindu \\ University, Varanasi, Uttar Pradesh, India \\ 2 Department of Obstetrics and Gynaecology, IMS, Banaras Hindu \\ University, Varanasi, Uttar Pradesh, India \\ Address for correspondence Pramod K. Singh, MD, Department of \\ Radiodiagnosis and Imaging, IMS, Banaras Hindu University, Varanasi, \\ Uttar Pradesh, India (e-mail: pramodk.singh1@bhu.ac.in).
}

Indian J Radiol Imaging 2022;32:10-15.

\begin{abstract}
Aim The aim of this article was to evaluate uterocervical angle (UCA) and cervical length $(\mathrm{CL})$ measured at 16 to 24 weeks of gestation using transvaginal sonography (TVS) as predictors of spontaneous preterm birth.

Methods In this prospective study, TVS was performed in 159 primigravidas with a singleton, uncomplicated pregnancy at 16 to 24 weeks of gestation to measure the anterior UCA and CL. All the cases were followed until labor to document gestational age at delivery.

Results The risk of spontaneous preterm birth was higher in women with obtuse UCA ( $>95$ degrees) with sensitivity of $86.7 \%$, specificity of $93.0 \%$, positive predictive value of $83.0 \%$, negative predictive value of $94.6 \%$, and $p$-value of $<0.001$. The difference between the means was statistically significant ( $p$-value $<0.001$ ). UCAs $\geq 105$ degrees and 95 to 105 degrees were found to be significantly associated with spontaneous preterm births at $<34$ weeks and 34 to 37 weeks, respectively. $\mathrm{CL}<2.5 \mathrm{~cm}$ was found to

\section{Keywords}

- uterocervical angle

- cervical length

- spontaneous preterm birth

- transvaginal sonography predict spontaneous preterm births at $<37$ weeks with sensitivity of $31.1 \%$, specificity of $95.6 \%$, and $p$-value of $<0.001$. UCA was found to be a better predictor of spontaneous preterm birth with a higher coefficient of variation $(56.4 \%)$ when compared with CL (16.9\%).

Conclusions UCA proved to be a novel ultrasound parameter that can serve as a better predictor of spontaneous preterm births in comparison to CL. A strong correlation exists between obtuse UCA and a risk of spontaneous preterm birth.
\end{abstract}

\section{Introduction}

Preterm birth (PTB) is defined as childbirth occurring at less than 37 completed weeks or 259 days of gestation and is a major determinant of neonatal mortality and morbidity. ${ }^{1}$ Given the substantial and far-reaching impact of PTB, it is important to recognize patients at increased risk. Evaluation of the cervix has been used as a tool to predict preterm labor based on the concept that the cervix acts as an anatomical marker of the underlying pathological process leading to spontaneous PTB (sPTB). ${ }^{2}$

The uterine cervix is under the effect of some pressures applied by surrounding pelvic organs and the growing uterus during pregnancy. Consequently, a combined effect of these published online

February 28, 2022
DOI https://doi.org/ $10.1055 / \mathrm{s}-0041-1741411$ ISSN 0971-3026.

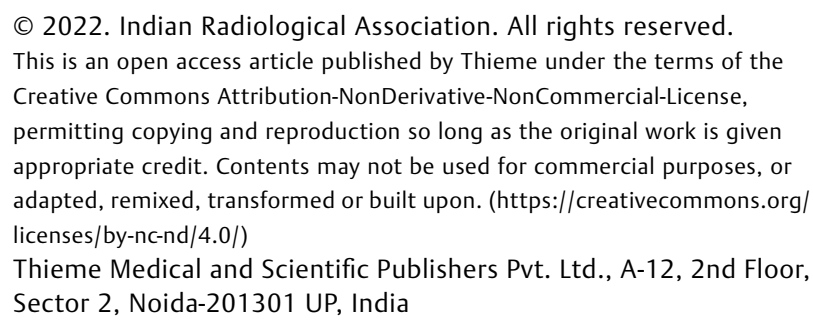


physiologic pressures and individual anatomy results in alteration of internal ostium (os) and cervical function. ${ }^{3}$ Some ultrasonographic parameters have been defined to assess the cervical structure such as cervical length (CL) and uterocervical angle (UCA). ${ }^{4}$

The ultrasound assessment of the cervix based on CL has been demonstrated to be a good predictor of preterm delivery during the first and second trimesters. ${ }^{5}$ However, the evidence of the effectiveness of universal second-trimester screening of $\mathrm{CL}$ is contradictory. ${ }^{6,7}$ Recently, the anterior UCA, the angle formed between the endocervical canal and anterior uterine wall, has been suggested as a predictor of sPTB. ${ }^{8,9}$

The pathophysiological principle behind the hypothesis is purely physical. The force that a gravid uterus transmits to the cervix differs depending on the anterior UCA. ${ }^{10}$ With advancing pregnancy, the force of the pregnant uterus is shifted toward the cervix and depending on the inclination angle, the cervical canal is either "pressed shut" in cases of an acute angle or "pressed open" in cases of an obtuse angle. A wide anterior UCA is thought to be associated with a more direct, linear outlet of uterine contents on internal os of cervix, and on the other hand, narrower UCA results in a lesser direct force on the internal os, which may be protective for cervical deformation. ${ }^{11}$

The present study was undertaken to evaluate the utility of the UCA and CL, measured using transvaginal sonography (TVS) during second trimester of pregnancy in prediction of spontaneous preterm labor.

\section{Methods}

\section{Subjects}

This prospective study was performed in the Department of Radiodiagnosis \& Imaging from January 2018 to June 2019. The study subjects were pregnant females with singleton, uncomplicated pregnancy who were referred for TVS at 16 to 24 weeks of gestation. The study was approved by institutional ethical committee at the outset and informed consent was obtained from all the patients. At the time of recruitment of these pregnant women into the study, all the data regarding clinical profile and relevant investigations were collected. The imaging was performed by a trainee radiologist (RS) in presence of and under the supervision of senior consultant radiologist with an experience of 10 years (PKS).

The inclusion criteria of this study consisted of (i) pregnant women between 19 and 34 years of age (ii) carrying singleton gestation. Patients were excluded if they had (i) age $<19$ years and >34 years; (ii) other maternal or fetal indications of preterm labor; (iii) multiple pregnancy; (iv) polyhydramnios; (iv) previous history of cervix surgery; (iv) systemic illnesses such as diabetes, thyroid disorders, hypertension, chronic renal disease; (v) previous history of PTBs; and (vi) uterine anomalies.

\section{Scan Procedure}

TVS examinations were performed in all pregnant women, under aseptic precautions, using high-frequency endovaginal probe (3-9 MHz) on a Philips iU22 ultrasound system (Philips Healthcare, Best, the Netherlands). The pregnant women were instructed to empty their bladder and were placed in dorsal lithotomy position. The vaginal probe was placed in anterior fornix without undue pressure. The sagittal view of the cervix and anterior uterine wall was obtained with optimal magnification. The echogenic endocervical canal was identified with surrounding hypoechoic zone of cervical mucosa. External os was visualized as the point where anterior and posterior lips of cervix come together in vaginal canal and internal os as the point where the cervical mucosa ends in lower uterine segment.

CL and UCA were measured using in-built angle caliper of our equipment. The anterior UCA is the angle between a line along the cervix and another line along the lower anterior uterine segment extending from internal os. Using angle caliper, two lines were drawn manually. The first line was drawn between internal and external os. The second line was drawn from the internal os along the lower aspect of anterior uterine wall to a point on the uterine wall $\sim 3 \mathrm{~cm}$ from the internal os. The anterior UCA was formed by the intersection of these two lines at internal os and recorded as per existing imaging protocol in the literature (-Fig. 1).$^{12}$ Using distance caliper, CL was measured along the echogenic endocervical

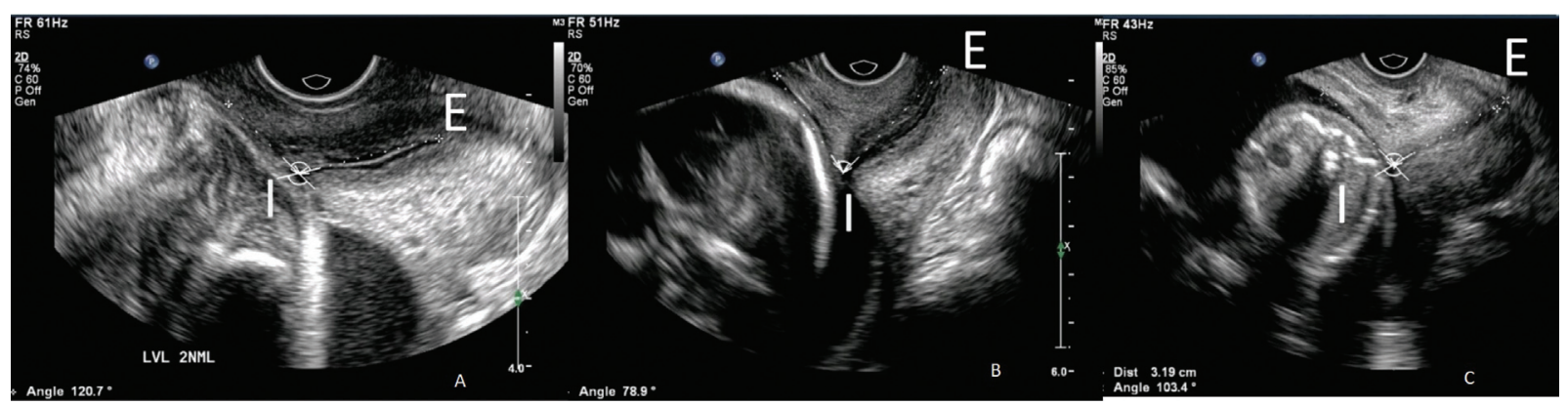

Fig. 1 Uterocervical angle (UCA). Transvaginal sonographic images showing technique of measurement of uterocervical angle. UCA was calculated as angle between two lines. The first line was drawn between internal (I) and external ostium (os) (E). The second line was drawn $3 \mathrm{~cm}$ parallel to the lower aspect of anterior inner uterine wall passing through the end of the first line at internal os. (A) Obtuse UCA $>105$ degrees in a pregnant woman (gestational age 19 weeks) who delivered preterm at $<34$ weeks. (B) Acute UCA $<95$ degrees in another pregnant woman (gestational age 22 weeks) who delivered at term (>37 weeks). (C) Obtuse UCA in the range 95 to 105 degrees in another pregnant woman (gestational age 22 weeks) who delivered preterm at 34 to 37 weeks. 


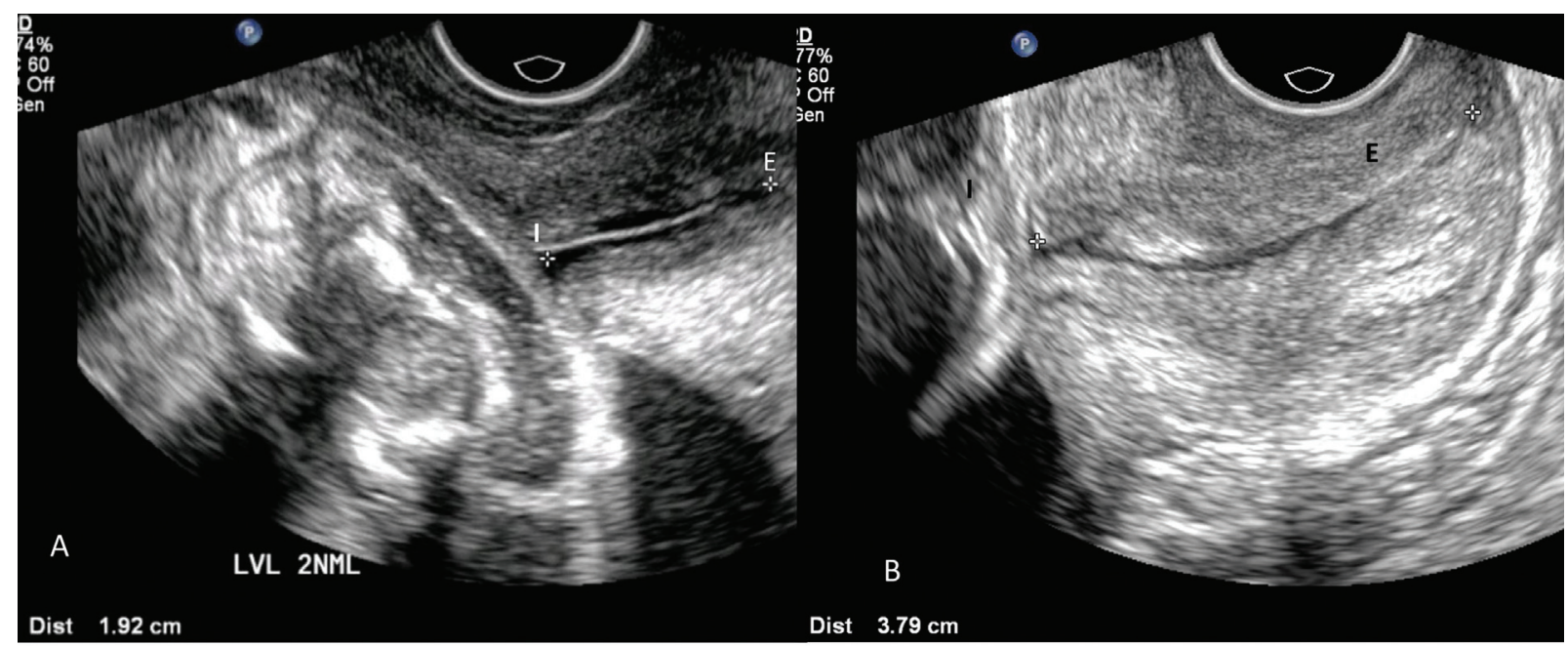

Fig. 2 Cervical length (CL). Transvaginal sonographic images showing technique of measurement of cervical length that was measured between internal os (I) and external os (E). (A) Higher $\mathrm{CL}(>2.5 \mathrm{~cm})$ in a pregnant woman of gestational age 20 weeks who delivered at term. (B) Short CL $(<2.5 \mathrm{~cm})$ in another pregnant woman (gestational age 19 weeks) who delivered preterm at $<37$ weeks.

canal from internal to the external cervical os. An average of three measures was taken (-Fig. 2).

\section{Clinical Follow-Up}

The pregnant women recruited in the study were closely followed up to delivery. Demographic and pregnancy outcome data were collected and recorded. PTB was defined as any birth before 37 weeks completed weeks of gestation. Maternal progesterone was given to all women with short CL $(<20 \mathrm{~mm}$ and more than $10 \mathrm{~mm}$ ) to support pregnancy. Cervical cerclage was not done in any of the cases.

\section{Statistical Analysis}

Statistical analysis was performed using SPSS software (Version 22.0. IBM Corp. Armonk, New York, United States). Student's $t$-test and analysis of variance was used to compare means between the two or more groups.

\section{Results}

Following the inclusion and exclusion criteria, 159 patients were included in our study, out of which, 45 women had sPTBs (18 underwent cesarean section after initiation of labor and 27 had normal delivery), whereas 114 women delivered at term (9 underwent cesarean section and 105 underwent spontaneous vaginal delivery). In the preterm group, the mean gestational age of delivery was 35.5 weeks.
Preterm group was further divided into two groups, based on time of delivery: (a) $<34$ weeks $(n=13)$ and (b) 34 to 37 weeks $(n=32)$.

Out of the 45 neonates with premature birth, 16 neonates required immediate admission in the neonatal intensive care unit, 6 neonates had 1 minute Apgar score $<7$, and 2 neonates died.

Obtuse UCA $\geq 95$ degrees was observed in 47 women out of which $39(83.0 \%)$ had PTBs ( $<37$ weeks) ( - Table 1). UCA in the second trimester was wider in the preterm group (103.7 \pm 8.62 degrees) compared with term group $(88.4 \pm 6.81$ degrees) ( $p$-value $<0.001)$. Further, within the preterm group, UCA was higher in the group with age $<34$ weeks (UCA $=$ $111.5 \pm 9.1$ degrees) than that of 34 to 37 weeks (UCA $=$ $100.4 \pm 6.0$ degrees), although the difference was not statistically significant. The incidence of SPTBs at $<34$ weeks was highest (84.6\%) in women with UCA of $\geq 105$ degrees and the incidence of SPTBs at 34 to 37 weeks was highest (78.1\%) in women with UCA of the range between 95 and 105 degrees (-Table 1). On receiver operating characteristic (ROC) curve analysis, we found that at an optimal cutoff point of 96 degrees, UCA $\geq 95$ degrees was a strong predictor of sPTBs ( $<37$ weeks) with area under the ROC curve being 0.926 ( - Fig. 3 ).

$\mathrm{CL}<2.5 \mathrm{~cm}$ was seen in 19 women, in which 14 had PTBs and 5 delivered at term ( $\mathbf{- T a b l e ~} 2$ ) The incidence of sPTBs was higher (73.7\%) in women with $\mathrm{CL}<2.5 \mathrm{~cm}$ than those with $\mathrm{CL} \geq 2.5 \mathrm{~cm}(22.1 \%)$. The mean $\mathrm{CL}$ was significantly

Table 1 Distribution of spontaneous preterm births (at $<37$ weeks) and term births in women according to uterocervical angle (UCA)

\begin{tabular}{|l|l|l|l|l|}
\hline \multicolumn{2}{|l|}{ UCA } & Delivery at $\geq \mathbf{3 7}$ wks & \multicolumn{2}{l|}{ Spontaneous preterm births $(<\mathbf{3 7}$ wks) } \\
\hline \multicolumn{2}{|l|}{$\geq 95$ degrees } & & Delivery at $<\mathbf{3 4}$ wks & Delivery at 34-37 wks \\
\cline { 2 - 5 } & $\mathbf{1 0 5}$ degrees & $1(0.9 \%)$ & $11(84.6 \%)$ & $2(6.2 \%)$ \\
\hline$<95$ degrees & $7(6.1 \%)$ & $1(7.7 \%)$ & $25(78.1 \%)$ \\
\hline
\end{tabular}

Pearson chi-square 21.901a; $p$-value $<0.001$. 


\section{ROC Curve}

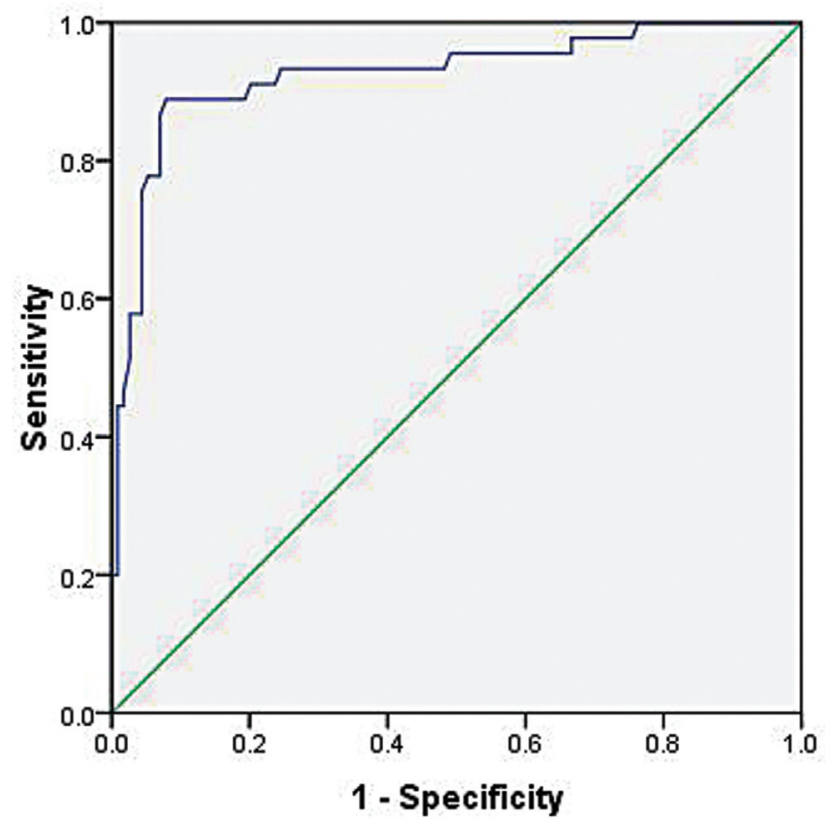

Diagonal segments are produced by ties.

Fig. 3 Receiver operating characteristic (ROC) plot for uterocervical angle with area under the ROC curve being 0.926 ( $p$-value $<0.001$, standard error: $\pm 0.026,95 \%$ confidence interval: $0.874-0.977$ ).

higher in pregnant women in term group ( $3.4 \pm 0.45)$ compared with preterm group $(3.1 \pm 0.69)(p$-value $<0.001)$ (-Table 2). However, the area under the ROC curve to predict preterm labor for CL was not significant with area under curve of 0.651 .

There was a significant negative correlation between UCA and gestational age at delivery $(r=-0.751 ; p$-value $<0.001)$ and significant positive correlation $(r=0.412 ; p$-value $<0.001$ ) between CL and gestational age at delivery. Linear
Table 2 Distribution of spontaneous preterm births (at $<37$ wks) and term births in women according to cervical length

\begin{tabular}{|l|l|l|}
\hline $\begin{array}{l}\text { Cervical } \\
\text { length }(\mathrm{cm})\end{array}$ & $\begin{array}{l}\text { Spontaneous } \\
\text { preterm births } \\
(<37 \text { wks) }\end{array}$ & $\begin{array}{l}\text { Term delivery } \\
(\geq 37 w k s)\end{array}$ \\
\cline { 2 - 3 } & $\mathbf{n ( \% )}$ & $\boldsymbol{n}(\%)$ \\
\hline$<2.5$ & $14(31.1 \%)$ & $5(4.4 \%)$ \\
\hline$\geq 2.5$ & $31(68.9 \%)$ & $109(95.6 \%)$ \\
\hline
\end{tabular}

Pearson chi-square 1.859 E2a; $p$-value $<0.001$.

regression analysis was done to evaluate the impact of the two predictor variables, that is, UCA and CL on the gestational age at delivery, which yielded a high coefficient of variation $\left(r^{2}\right)$ for UCA (0.564) ( - Fig. 4A) than CL (0.169) (-Fig. 4B) (-Table 3). Spearman's correlation coefficient between the two testing parameters UCA and CL was found to be -0.264 ( $p$-value $<0.001)$.

\section{Discussion}

PTB is associated with an increased risk of neonatal mortality and morbidity. ${ }^{13}$ SPTB is thought to originate from various factors including weakness in cervical tissue that otherwise act as a barrier between uterus and vagina.

The mechanical strength of cervix relies on the strength of collagen network. ${ }^{14}$ Cervical incompetence is caused due to premature cervical softening, a process that reduces cervical compliance and resistance to deformation through disarrangement of collagen fibrils and a $5 \%$ increase in tissue hydration. $^{3,15}$

Assessment of cervix has been done using various techniques such as digital examination of cervix, TVS, cervical elastography, and quantification of cervical apparent diffusion coefficient (ADC). Digital examination underestimates CL by a mean difference of $12 \mathrm{~mm}$ in more than $80 \%$ of women in the second and third trimesters compared with
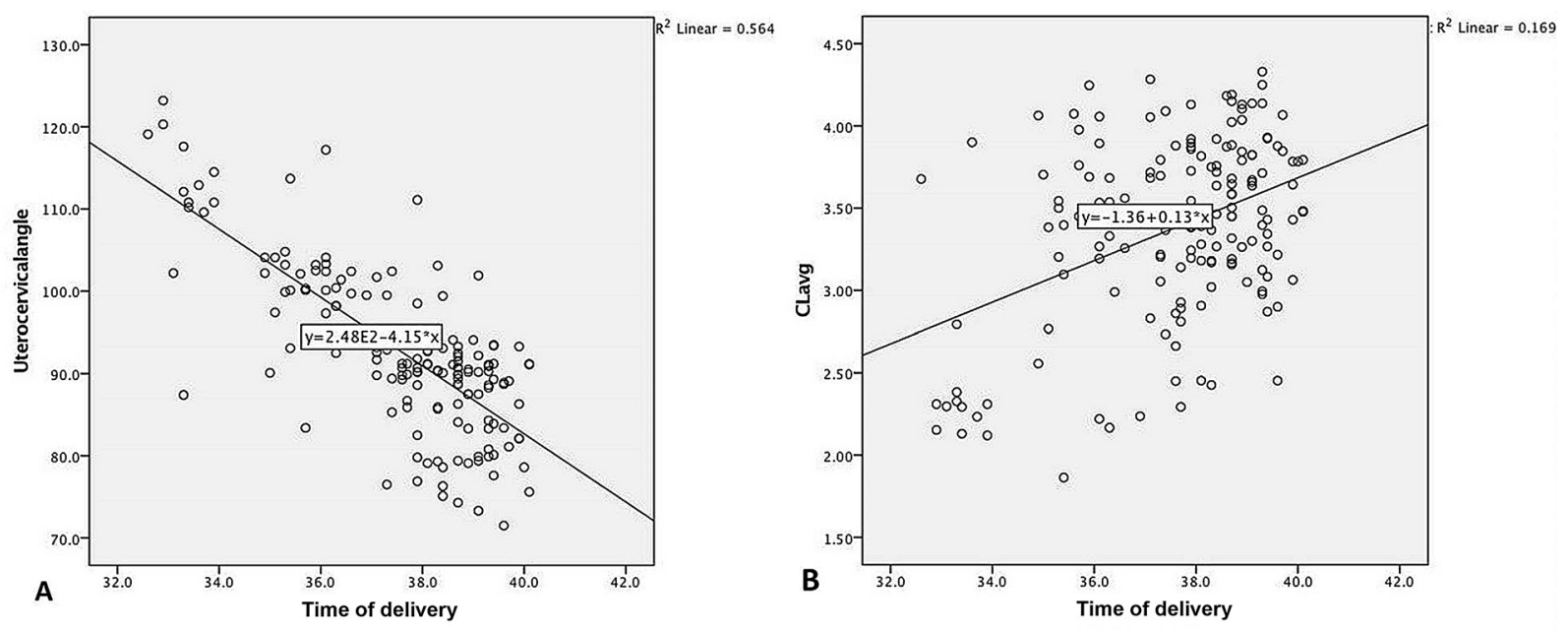

Fig. 4 Regression analysis: (A) Scatter plot between uterocervical angle and gestational age at delivery showing statistically significant negative correlation between them with $r^{2}=0.564$. (B) Scatter plot between cervical length and gestational age at delivery showing statistically significant positive correlation between them with $r^{2}=0.16$. 
Table 3 Regression analysis for uterocervical angle and cervical length

\begin{tabular}{|l|l|l|l|}
\hline Parameter & Beta value & $\begin{array}{l}\text { Coefficient of } \\
\text { variation, } r^{2}\end{array}$ & $p$-Value \\
\hline $\begin{array}{l}\text { Uterocervical } \\
\text { angle }\end{array}$ & -0.136 & 0.564 & $<0.001$ \\
\hline Cervical length & 1.344 & 0.169 & $<0.001$ \\
\hline
\end{tabular}

sonography. TVS is an excellent tool for the assessment of the cervical anatomy. CL measured by TVS is mostly used in obstetrics as a screening test for the prediction of PTB. A shortened cervix $<2.5 \mathrm{~cm}$ has been identified as an important indicator of preterm labor. ${ }^{16}$ It is thought to be caused due to proximal cervical effacement in which muscle fibers located at the internal os are incorporated into lower uterine segment. ${ }^{1}$ However, some authors suggest that shortened cervix may be simply a sign that accompanies some of the abnormal processes leading to $\mathrm{SPTB}$, rather than a cause in itself.

In this study, $\mathrm{CL}<2.5 \mathrm{~cm}$ was found to be associated with sPTB at $<37$ weeks with a low sensitivity of $31.1 \%$ and a specificity of $95.6 \%$ (-Table 2). This is in agreement with observations made by Iams et $\mathrm{al}^{17}$ who reported that CL $<2 \mathrm{~cm}$ at 24 weeks has a sensitivity of $23 \%$, and a specificity of $97 \%$ for PTB before 35 weeks in a population with a SPTB rate of $4.3 \%$. Similar observations were made by Romero et al, ${ }^{18}$ Sur et $\mathrm{al}^{19}$ and Boelig et $\mathrm{al}^{20}$ in their respective studies. Various studies have reported low sensitivity (60\%) and up to $10 \%$ false-positive rate for $\mathrm{CL}$ in prediction of preterm labor. ${ }^{21}$

Recently, the potential of UCA as an ultrasound marker to predict $\mathrm{SPTB}$ has been identified. ${ }^{22} \mathrm{~A}$ wide UCA detected during the second trimester is associated with an increased risk of spontaneous preterm labor. The rationale behind this association is based on the mechanical properties of the UCA. An acute UCA acts as a preventive mechanical barrier against sPTB. ${ }^{23}$

In this study, UCA in the second trimester was significantly wider in the preterm group compared with the term group and there was a significant association between obtuse UCA and risk of spontaneous preterm labor. We found UCA $\geq 105$ degrees to be significantly associated with SPTBs at $<34$ weeks. UCA in the range 95 to 105 degrees was found to be significantly associated with SPTBs at 34 to 37 weeks with a sensitivity of $78.1 \%$, specificity of $93.7 \%$, and $p$-value $<0.001$ (-Table 2) This is in concordance with previous study by Dziadosz et al. ${ }^{9}$ We hypothesize that the cervical softening impedes the ability of proximal cervical fibers to retain an acute angle at the internal os, consistent with observation of our study and various previous studies that an obtuse angle is associated with sPTB. ${ }^{8,19,24}$ Also, the obtuse angle may represent the precursor stage of incorporation of internal os as an anatomical and physiological part of lower uterine segment.

UCA was found to be more sensitive in prediction of SPTBs than that of $\mathrm{CL}(31.1 \%)$. This finding is in agreement with the findings of a previous studies. ${ }^{9}$ Lynch et $\mathrm{al}^{24}$ compared performance metrics of UCA with CL and observed that
UCA was accompanied by significant sensitivity (exceeding $80 \%$ ) but low specificity $(<35 \%)$, whereas CL was more specific (98.5\%) but less sensitive (12.5\%). Knight et $\mathrm{al}^{25}$ also evaluated second-trimester UCA and observed that the use of optimal cutoffs resulted in enhanced predictive accuracy compared with CL measurement $(<20 \mathrm{~mm})$ for PTB $<34$ weeks.

In this study, we found a statistically significant negative correlation between UCA and gestational age at delivery $(r=-0.751)$. This implied that as the UCA becomes obtuse ( $>95$ degrees), the incidence of sPTBs increases. On comparison of the two predictor variables using regression analysis, the coefficient of variation of UCA was higher (56.4\%) than CL (16.9\%). Henceforth, UCA proved to be stronger predictor of sPTBs than that of $\mathrm{CL}$.

Several factors such as ethnicity, race, and sociodemographic conditions of the patients can potentially affect cervical properties including CL and UCA. Studies have demonstrated that there are racial discrepancies of second trimester short CL in black women compared with white, Asian and Hispanic women. ${ }^{26}$ A study by Thain et al found a significantly shorter median CL at 28 to 22 weeks and at 28 to 32 weeks in the Indian population compared with the other ethnicities. ${ }^{27}$ To the best of our knowledge, no study has been undertaken to evaluate the relation of ethnicity and UCA.

Timely recognition of pregnant women at increased risk of preterm labor can facilitate timely interventions for its management such as surgical closure of cervix with cervical cerclage, maternal progesterone supplementation, and pessaries.

Previous studies have shown that progesterone treatment may prevent PTB in women known to have a short cervix on ultrasound imaging in mid-pregnancy. ${ }^{16}$ The American College of Obstetricians and Gynecologists recommends vaginal progesterone for women with incidental short cervix, CL $<20 \mathrm{~mm}$ but more $>10 \mathrm{~mm}$. The results of our studies highlight the need of revision of the existing recommendations. Our study supports the introduction of UCA as a factor to initiate progesterone administration in these women. The current available evidence does not support cerclage placement in women with sonographic short cervix without history of sPTB. ${ }^{25}$

We realize that our study had few limitations. First our study had a small sample size. Second, in patients with high UCA, we did not actually evaluate the impact of progesterone administration on its ability to prevent SPTB. Moreover, patients with CL less than $20 \mathrm{~mm}$ were given progesterone that can act as a confounding factor. The strength of this study was its prospective nature. Advanced imaging techniques such as cervical elastography and measurement of subglandular ADC on magnetic resonance imaging may be further undertaken in the future to predict SPTB.

\section{Conclusion}

Our study suggests that the UCA is a potential novel screening tool for the prediction of SPTB. UCA is a better predictor of SPTB than CL. However, detection rates of SPTB can further be 
improved if combined with other parameters such as CL, maternal demographic and obstetric history. This would be of immense importance in early prediction of SPTB to facilitate timely interventions for its optimum management, which may lead to better neonatal outcomes.

\section{Conflict of Interest}

None.

\section{References}

1 Cunningham FG, Leveno KG, Bloom SL, Spong CY. Preterm labor. In: Cunningham F, Leveno KG, Bloom SL, Spong CY, eds. William Obstetrics. 24th edition. New York: McGraw-Hill Education/ Medical; 2014;42:829-830

2 Wendy LW, Katherine WF. Cervical ultrasound and preterm birth. In: Rumack CM, Wilson SR, Charboneau W, Levine D, eds. Diagnostic Ultrasound. 4th edition. Philadelphia: Elsevier Mosby; 2011;45:1527-1528

3 House M, McCabe R, Socrate S. Using imaging-based, threedimensional models of the cervix and uterus for studies of cervical changes during pregnancy. Clin Anat 2013;26(01): 97-104

4 Myers KM, Feltovich H, Mazza E, et al. The mechanical role of the cervix in pregnancy. J Biomech 2015;48(09):1511-1523

5 Greco E, Gupta R, Syngelaki A, Poon LC, Nicolaides KH. Firsttrimester screening for spontaneous preterm delivery with maternal characteristics and cervical length. Fetal Diagn Ther 2012; 31(03):154-161

6 Son M, Grobman WA, Ayala NK, Miller ES. A universal midtrimester transvaginal cervical length screening program and its associated reduced preterm birth rate. Am J Obstet Gynecol 2016;214(03):365.e1-365.e5

7 Temming LA, Durst JK, Tuuli MG, et al. Universal cervical length screening: implementation and outcomes. Am J Obstet Gynecol 2016;214(04):523.e1-523.e8

8 Sochacki-Wójcicka N, Wojcicki J, Bomba-Opon D, Wielgos M. Anterior cervical angle as a new biophysical ultrasound marker for prediction of spontaneous preterm birth. Ultrasound Obstet Gynecol 2015;46(03):377-378

9 Dziadosz M, Bennett TA, Dolin C, et al. Uterocervical angle: a novel ultrasound screening tool to predict spontaneous preterm birth. Am J Obstet Gynecol 2016;215(03):376.e1-376.e7

10 Sepúlveda-Martínez A, Díaz F, Muñoz H, Valdés E, Parra-Cordero $M$. Second-trimester anterior cervical angle in a low-risk population as a marker for spontaneous preterm delivery. Fetal Diagn Ther 2017;41(03):220-225

11 Farràs Llobet A, Higueras T, Calero IZ, et al. Prospective evaluation of the uterocervical angle as a predictor of spontaneous preterm birth. Acta Obstet Gynecol Scand 2020;99(11):1511-1518

12 Llobet F, Reginc'os, Higueras T. The utero cervical angle and its relationship with pre term birth. J Matern Fetal Neonatal Med 2017;31(14):1881-1884
13 Spong CY. Prediction and prevention of recurrent spontaneous preterm birth. Obstet Gynecol 2007;110(2 Pt 1):405-415

14 Yoshida K, Jiang H, Kim M, et al. Quantitative evaluation of collagen crosslinks and corresponding tensile mechanical properties in mouse cervical tissue during normal pregnancy. PLoS One 2014;9(11):e112391

15 Myers K, Socrate S, Tzeranis D, House M. Changes in the biochemical constituents and morphologic appearance of the human cervical stroma during pregnancy. Eur J Obstet Gynecol Reprod Biol 2009;144(Suppl 1):S82-S89

16 Sonek JD, Iams JD, Blumenfeld M, Johnson F, Landon M, Gabbe S. Measurement of cervical length in pregnancy: comparison between vaginal ultrasonography and digital examination. Obstet Gynecol 1990;76(02):172-175

17 Iams JD, Goldenberg RL, Meis PJ, et al; National Institute of Child Health and Human Development Maternal Fetal Medicine Unit Network. The length of the cervix and the risk of spontaneous premature delivery. N Engl J Med 1996;334(09):567-572

18 Romero R, Nicolaides K, Conde-Agudelo A, et al. Vaginal progesterone in women with an asymptomatic sonographic short cervix in the midtrimester decreases preterm delivery and neonatal morbidity: a systematic review and metaanalysis of individual patient data. Am J Obstet Gynecol 2012;206(02):124.e1-124.e19

19 Sur B, Misra S, Dash S. Evaluation of the anterior cervical angle of the uterus to predict spontaneous preterm birth. Int J Reprod Contracept Obstet Gynecol 2017;6:2323-2327

20 Boelig RC, Orzechowski KM, Berghella V. Cervical length, risk factors, and delivery outcomes among women with spontaneous preterm birth. J Matern Fetal Neonatal Med 2016;29(17): 2840-2844

21 Celik E, To M, Gajewska K, Smith GC, Nicolaides KHFetal Medicine Foundation Second Trimester Screening Group. Cervical length and obstetric history predict spontaneous preterm birth: development and validation of a model to provide individualized risk assessment. Ultrasound Obstet Gynecol 2008;31(05):549-554

22 Dagdeviren E, Aslan Çetin B, Aydogan Mathyk B, Koroglu N, Topcu EG, Yuksel MA. Can uterocervical angles successfully predict induction of labor in nulliparous women? Eur J Obstet Gynecol Reprod Biol 2018;228:87-91

23 Daskalakis G. Assessment of uterocervical angle width as a predictor of preterm birth: a systematic review of literature. BioMed Res Int 2018;18:374-378

24 Lynch TA, Szlachetka K, Seligman NS. Ultrasonographic change in uterocervical angle is not a risk factor for preterm birth in women with a short cervix. Am J Perinatol 2017;34(11):1058-1064

25 Knight JC, Tenbrink E, Onslow M, Patil AS. Uterocervical angle measurement improves prediction of preterm birth in twin gestation. Am J Perinatol 2018;35(07):648-654

26 Buck JN, Orzechowski KM, Berghella V. Racial disparities in cervical length for prediction of preterm birth in a low risk population. J Matern Fetal Neonatal Med 2017;30(15):1851-1854

27 Thain S, Yeo GSH, Kwek K, Chern B, Tan KH. Spontaneous preterm birth and cervical length in a pregnant Asian population. PLoS One 2020;15(04):e0230125 